Revue musicale OICRM

\title{
Debussy saisi par les Indépendants. Une contribution à l'analyse du processus de reconnaissance de l'oeuvre de Debussy
}

\section{Alexandre Robert}

Volume 2, numéro 1, 2014

La réception de Debussy au XXe siècle. Incidences, influences et autorité

URI : https://id.erudit.org/iderudit/1055850ar

DOI : https://doi.org/10.7202/1055850ar

Aller au sommaire du numéro

Éditeur(s)

OICRM

ISSN

2368-7061 (numérique)

Découvrir la revue

Citer cet article

Robert, A. (2014). Debussy saisi par les Indépendants. Une contribution à l'analyse du processus de reconnaissance de l'oeuvre de Debussy. Revue musicale OICRM, 2(1), 141-158. https://doi.org/10.7202/1055850ar

\section{Résumé de l'article}

Comment l'oeuvre de Debussy en est-il venu à être considéré comme l'un des plus importants de l'histoire de la musique occidentale ? Il est certain que le processus de reconnaissance auquel on a ici affaire est d'une redoutable complexité. Néanmoins, cet article se propose d'isoler et d'examiner une des étapes cruciales, à savoir la reconnaissance de Debussy par certains de ses pairs. Notre thèse est la suivante : la reconnaissance dont jouit aujourd'hui l'œuvre de Debussy est largement tributaire de son appropriation, dans les années 1900 et 1910, par les musiciens français qui constituent le groupe des « Indépendants ». Il semble indispensable, dans un premier temps, d'élargir l'échelle d'observation et de restituer la position collective des Indépendants au sein de la structure de l'univers macro-social relativement autonome qu'est le " champ musical français » du début du XXe siècle. On peut alors montrer qu'ils s'inscrivent dans une double opposition - indissociablement institutionnelle, esthétique et musicale - à l'Institut et surtout à la Schola Cantorum de Vincent d'Indy. Dans ce contexte de lutte, un examen des écrits d'Émile Vuillermoz, Florent Schmitt, Maurice Ravel et Charles Koechlin montre que les

Indépendants " saisissent »-au double sens du terme, c'est-à-dire " s'emparent de " et " comprennent "- l'œuvre de Debussy au travers de logiques esthétiques spécifiques (logique de l'émotion et logique de l'innovation). Si l'appropriation de l'oeuvre de Debussy par les Indépendants est si décisive, ce n'est pas seulement parce qu'ils sont parmi les premiers à en faire un jalon indiscutable de l'histoire de la musique ; c'est aussi parce qu'ils s'en réclament de façon cohérente et quasi organisée pour fonder leur propre existence artistique. C'est par cette double action, qui consiste à la fois à faire de l'auteur de Pelléas le père fondateur de leur lignée artistique et à lutter (avec succès) pour exister au sein du champ musical français, que les Indépendants font grandir peu à peu l'oeuvre debussyste.
Ce document est protégé par la loi sur le droit d'auteur. L'utilisation des services d’Érudit (y compris la reproduction) est assujettie à sa politique d'utilisation que vous pouvez consulter en ligne.

https://apropos.erudit.org/fr/usagers/politique-dutilisation/ 


\title{
Debussy saisi par les Indépendants. Une contribution à l'analyse du processus de reconnaissance de l'oeuvre de Debussy
}

\author{
Alexandre Robert
}

\begin{abstract}
Résumé
Comment l'œuvre de Debussy en est-il venu à être considéré comme l'un des plus importants de l'histoire de la musique occidentale ? Il est certain que le processus de reconnaissance auquel on a ici affaire est d'une redoutable complexité. Néanmoins, cet article se propose d'isoler et d'examiner une des étapes cruciales, à savoir la reconnaissance de Debussy par certains de ses pairs. Notre thèse est la suivante : la reconnaissance dont jouit aujourd'hui l'œuvre de Debussy est largement tributaire de son appropriation, dans les années 1900 et 1910, par les musiciens français qui constituent le groupe des "Indépendants". Il semble indispensable, dans un premier temps, d'élargir l'échelle d'observation et de restituer la position collective des Indépendants au sein de la structure de l'univers macro-social relativement autonome qu'est le " champ musical français » du début du $\mathrm{xx}^{\mathrm{e}}$ siècle. On peut alors montrer qu'ils s'inscrivent dans une double opposition - indissociablement institutionnelle, esthétique et musicale - à l'Institut et surtout à la Schola Cantorum de Vincent d'Indy. Dans ce contexte de lutte, un examen des écrits d'Émile Vuillermoz, Florent Schmitt, Maurice Ravel et Charles Koechlin montre que les Indépendants " saisissent » - au double sens du terme, c'est-à-dire " s'emparent de » et « comprennent »-l'œuvre de Debussy au travers de logiques esthétiques spécifiques (logique de l'émotion et logique de l'innovation). Si l'appropriation de l'œuvre de Debussy par les Indépendants est si décisive, ce n'est pas seulement parce qu'ils sont parmi les premiers à en faire un jalon indiscutable de l'histoire de la musique ; c'est aussi parce qu'ils s'en réclament de façon cohérente et quasi organisée pour fonder leur propre existence artistique. C'est par cette double action, qui consiste à la fois à faire de l'auteur de Pelléas le père fondateur de leur lignée artistique et à lutter (avec succès) pour exister au sein du champ musical français, que les Indépendants font grandir peu à peu l'œuvre debussyste.
\end{abstract}

Mots clés : appropriation ; champ musical ; Debussy ; Indépendants ; Schola Cantorum.

\begin{abstract}
How does Debussy's work became to be considered as one of the most important of occidental music history? It is certain that we here dealing with a very complex process of recognition. However, this article aims to isolate from it and to examine
\end{abstract}


one of its main stages, such as the recognition of Debussy by some of his peers. Our thesis is the following: the recognition that today knows Debussy's work is widely due to its appropriation between the 1900s and 1910s by French musicians from the group of the "Indépendants." It seems essential, in the first instance, to widen the scale of observation and restore the collective position occupied by this group within the structure of the relatively autonomous macro-social world that constitutes the "French musical field" of the early 20th century. We can then show that they are part of a dual opposition - inextricably institutional, aesthetic and musical - to the Institut and to the Schola Cantorum managed by Vincent d'Indy. In this conflicting context, an accurate review of the writings from Émile Vuillermoz, Florent Schmitt, Maurice Ravel and Charles Koechlin shows that the Indépendants take hold of Debussy's work through specific aesthetic logics (logic of emotion and logic of innovation). If this appropriation of Debussy's work by the Indépendants is so decisive, it is not only because they are among the first to make an indisputable milestone of history of music, but also because they identify with it in a coherent and nearorganized way to found their own artistic existence. It is through this double action, which consists on making of the author of Pelléas the founder of their artistic lineage and struggling (successfully) to exist within french musical field, that the Indépendants gradually make the value of Debussy's work grow.

Keywords: appropriation; Debussy; Indépendants; musical field; Schola Cantorum.

Comment la figure de Debussy s'est-elle imposée ? Comment son œuvre en est-il venu à être considéré comme l'un des plus marquants de l'histoire de la musique occidentale ? Il est certain que le processus de reconnaissance auquel on a affaire ici est d'une redoutable complexité, tant par son étendue temporelle - de la fin du $\mathrm{XIX}^{\mathrm{e}}$ siècle à aujourd'hui - que géographique - Debussy étant l'un des compositeurs français les plus célébrés à l'échelle internationale. Néanmoins, nous nous proposons ici d'isoler et d'examiner une des étapes cruciales qui y ont conduit, à savoir la reconnaissance de Debussy par certains de ses " pairs ${ }^{1}$ ". Notre thèse est la suivante : la reconnaissance dont jouit aujourd'hui l'œuvre de Debussy est en partie tributaire de son appropriation, dans les années 1900 et 1910, par les musiciens français qui constituent le groupe des «Indépendants ${ }^{2}$ » (Duchesneau 1997).

Nous montrerons dans un premier temps quelle position collective occupe à cette période le groupe des Indépendants au sein du " champ musical français ", puis nous examinerons la manière dont Debussy est peu à peu désigné fondateur de leur " lignée » artistique. Enfin, en examinant les écrits de Charles Koechlin, Maurice Ravel, Émile Vuillermoz et Florent Schmitt, nous verrons selon quelles catégories de perception l'œuvre de Debussy y est durablement érigée comme modèle.

1 Pour l'historien de l'art anglais Alan Bowness, « la reconnaissance des pairs est la première et à maints égards la plus significative» dans la construction du succès d'un artiste (Bowness [1989]2011, p. 13).

2 Notons qu'il s'agit en même temps d'un terme "indigène "; il est par exemple employé par Émile Vuillermoz en 1914 au détour d'une de ses critiques musicales (Vuillermoz 1914). 


\section{LA DYNAMIQUE DU CHAMP MUSICAL FRANÇAIS ENTRE 1890 ET 1918}

On ne peut réellement comprendre les logiques de l'appropriation de l'œuvre de Debussy par les Indépendants si l'on n'en restitue pas les conditions objectives et historiques de possibilité, c'est-à-dire si l'observation reste bloquée sur les seules actions et discours de ce groupe de musiciens. Il semble donc indispensable, en premier lieu, de porter en " arrière-plan » un regard sur l'univers macro-social relativement autonome que constitue la vie musicale française de l'époque et dans lequel les Indépendants sont " pris ", au sens où leurs actions y sont partiellement déterminées.

\section{Le concept de champ musical}

Le concept relationnel de «champ », proposé par Pierre Bourdieu (Bourdieu 1979, p. 249-288; Bourdieu [1992]1998), permet d'alimenter une telle démarche. Un champ est avant tout une sphère d'activité particulière traversée par des conflits, un réseau de relations objectives entre des positions occupées par des producteurs culturels, et notamment, dans notre perspective, par des compositeurs. Il s'agit ainsi d'un univers social spécifique doté d'une autonomie relative, c'est-à-dire de ses logiques propres, ses valeurs, ses règles du jeu et ses limites, lesquelles se redéfinissent sans cesse puisqu'elles sont l'enjeu de luttes et de guerres symboliques entre les individus qui occupent les positions. Il se caractérise donc (à un moment donné) par sa structure, mais aussi par son histoire et son dynamisme.

Ce puissant modèle théorique, "par le caractère systématique de sa visée et par le recours à une conceptualisation unitaire qui le rend applicable à tout secteur de la production symbolique " (Fabiani 1993, p. 152), a fait l'objet de nombreuses appropriations, dans des domaines divers tels que la poésie (Bandier 1999), la bandedessinée (Boltanski 1975), la peinture (Gamboni 1989), etc. Or, en dépit de limites historiques et théoriques certaines (Lahire [1999]2001 ; Fabiani [1999]2001), son usage nous semble tout indiqué pour appuyer notre argumentation. En effet, par un élargissement de la focale d'observation, il permet d'éclairer les luttes indissociablement institutionnelles, esthétiques et musicales qui structurent et dynamisent l'espace spécifique des compositeurs français entre 1890 et 1918, espace au sein duquel se négocie en partie la valeur des œuvres et des créateurs.

\section{Les années 1890 et la prise de pouvoir de d'Indy}

La vie musicale française de la fin du XIX siècle est indéniablement marquée par la création de la Société nationale de musique $^{3}$ en 1871 (Duchesneau 1997 ; Strasser 1998). Si son but premier était d'encourager la création - notamment instrumentale - chez les jeunes compositeurs français par l'organisation de concerts, elle a surtout eu pour effet d'amorcer la conquête d'une relative autonomie du champ 
musical français en créant un espace de décision esthétique libéré de l'influence et du contrôle de l'Institut - c'est-à-dire, en dernière instance, de l'État.

À l'orée des années 1890, le champ musical français se retrouve ainsi divisé, ou plutôt étiré entre deux pôles dominants. Il présente une structure duale, partagée entre deux principes de création opposés, fondée sur la double nature symbolique et économique des œuvres musicales. À l'instar du champ littéraire tel que le décrit Pierre Bourdieu, il est :

Le lieu de la coexistence antagoniste de deux modes de production et de circulation obéissant à des logiques inverses. À un pôle, l'économie anti" économique » de l'art pur, fondée sur la reconnaissance obligée des valeurs du désintéressement et sur la dénégation de 1' "économie » (du commercial) et du profit " économique " (à court terme), privilégie la production et ses exigences spécifiques, issues d'une histoire autonome [...]. À l'autre pôle, la logique "économique » [...] qui, faisant du commerce des biens culturels un commerce comme les autres, confère la priorité à la diffusion, au succès immédiat et temporaire, mesuré par exemple au tirage [et aussi, pour nous, au nombre d'exécution des œuvres, notamment des opéras], et se contente de s'ajuster à la demande préexistante de la clientèle [...] (Bourdieu [1992]1998, p. 235-236).

Dans cette présentation schématique, Vincent d'Indy incarne la figure dominante du pôle de création " pure " et de l'avant-garde musicale française. Il devient en effet président de la SNM en 1890, à la mort de César Franck, s'opposant aux compositeurs de l'Académie des Beaux-Arts tels que Massenet, Ernest Reyer, Saint-Saëns, Théodore Dubois ou Émile Paladilhe, représentants du pôle de la consécration institutionnelle et du profit économique, donc de l'arrière-garde. Bien sûr, ces deux pôles ne représentent que des tendances extrêmes, aucun compositeur ne pouvant être entièrement réduit à telle ou telle logique de création (voir figure 1).

L'opposition de d'Indy à l'Institut se manifeste parfois ouvertement et publiquement, à l'image d'un article paru en 1895 dans Le Figaro dans lequel il dénonce "l'inutilité flagrante » et "l'influence des plus délétères sur l'enseignement artistique [français] » (d'Indy 1895) qu'exerce le Prix de Rome, symbole de l'emprise étatique sur les carrières des jeunes compositeurs. En 1912, son rejet de l'Institut sera toujours aussi violent, l'amenant à préciser son point de vue dans Le Temps :

Être de l'Institut pour la notoriété ou la gloire que ce titre procure, cela ne me séduit aucunement. Je ne suis pas sensible à ce plaisir-là. [...] le destin naturel de l'artiste est de faire son œuvre ou ses œuvres (d'Indy 1912a).

On voit bien ici comment la légitimité institutionnelle est rejetée au profit d'une légitimité spécifique que le compositeur tire de sa volonté d'autonomisation du musical vis-à-vis de l'État. 


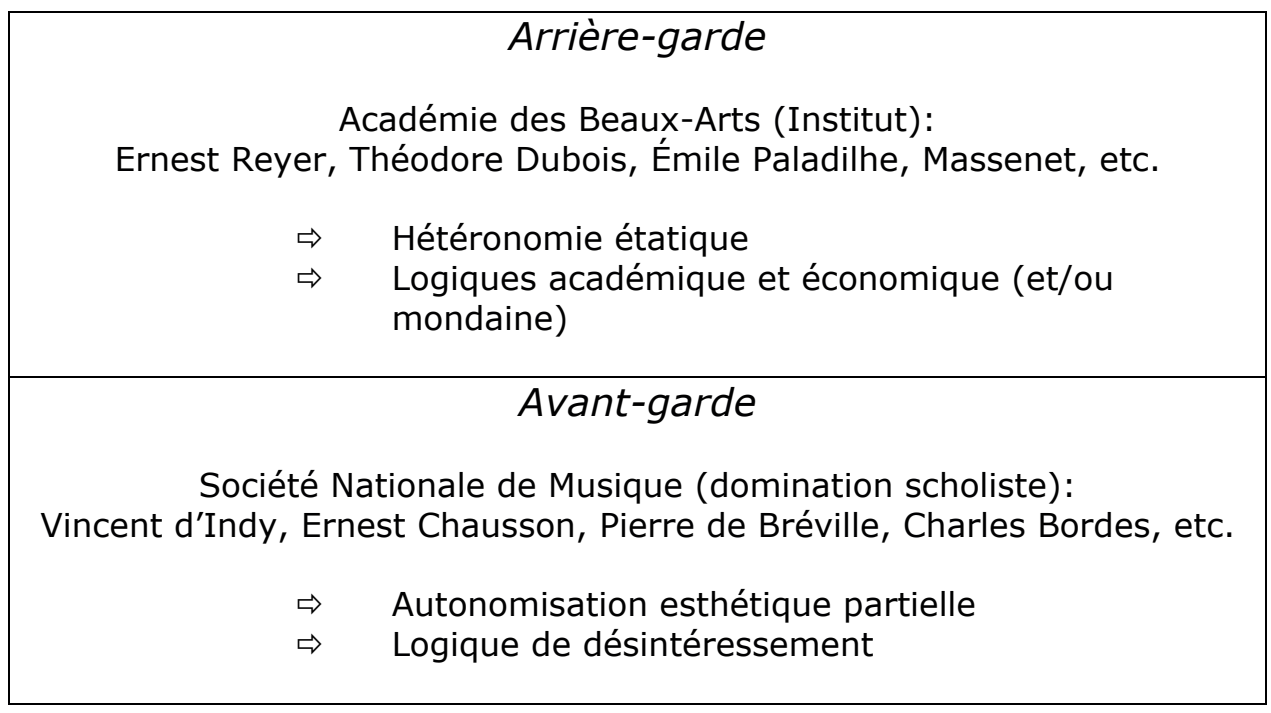

Figure 1 : Structure du champ musical français des années 1890.

La «scholisation » de l'avant-garde

La domination de Vincent d'Indy au sein de l'avant-garde du champ s'accentue considérablement avec la création de la Schola Cantorum. Cette école religieuse de musique est d'abord fondée en 1894 par Charles Bordes, Alexandre Guilmant et Vincent d'Indy, mais ce dernier, d'abord professeur de composition, en devient rapidement directeur. Progressivement, la Société nationale de musique se «scholise » :

Une analyse des archives et de la programmation de la sN révèle que [...] les éléments scholistes y sont de plus en plus nombreux. Louis de Serres, Pierre de Bréville, Pierre Coindreau, Gustave Bret, Déodat de Séverac seront membres du comité et remplaceront peu à peu les membres dont la neutralité était plus grande (Dukas, Erlanger, Lazzari) (Duchesneau 1997, p. 35).

Une sorte de monopole scholiste commence ainsi à se mettre en place au sein du comité de direction et de programmation de la SNM. Pour bien comprendre ce qu'implique cette emprise sur le plan esthétique, il convient de faire un détour par les écrits de Vincent d'Indy ${ }^{4}$. La lecture du Cours de composition musicale, par exemple, révèle une pensée esthétique indiscutablement subordonnée à un puissant ethos catholique. Il suffit, pour s'en convaincre, d'en citer l'introduction :

Sans la Foi, il n'est point d'Art [...]. L'Art est un, en soi ; seule l'expression, la manifestation diffère suivant le procédé employé par l'artiste pour l'exprimer. La raison de cette unité de l'Art est d'ordre surnaturel : au-dessus de tous les

4 La pensée esthétique de Vincent d'Indy a récemment fait l'objet de travaux historiques et musicologiques. Voir notamment l'ouvrage collectif dirigé par Manuela Schwartz, Vincent d'Indy en son temps (2006) et la thèse de Gilles Saint-Arroman, "Les écrits de Vincent d'Indy, d'une pensée à sa mise en acte » (2011). 
besoins humains plane l'aspiration vers la Divinité, l'élan de la créature vers son Auteur; et c'est dans l'Art, sous toutes ses formes, que l'âme cherche le moyen de rattacher sa vie à l'Etre qui en est le principe [...]. L'idée de l'Art nous apparaît donc, dès l'origine, indissolublement liée à l'idée religieuse, à l'adoration ou au culte divin (d'Indy 1912b, p. 10-11).

Toute la pensée musicale de Vincent d'Indy est ainsi explicitement structurée par des schèmes et des principes religieux, dont les plus saillants sont sans doute l'attachement à la tradition ou aux règles et 1'ascétisme de la pratique musicale ${ }^{5}$. Le "scholisme " peut donc être considéré comme un programme artistique qui, unifié par la figure dominante de d'Indy, relie pratique musicale et pratique religieuse, esthétique et éthique catholique.

Si la création musicale française avait en partie réussi à se défaire de la domination de l'Institut avec la fondation et l'activité de la SNM, l'action scholiste contribue peu à peu à réintroduire, au sein de l'avant-garde du champ, un nouveau principe d'hétéronomie en vertu duquel la musique se retrouve assujettie au religieux, l'esthétique subordonnée à l'éthique.

\section{Les années 1900 et la fracture de l'avant-garde}

À la toute fin des années 1890 et surtout au début des années 1900, la structure du champ commence imperceptiblement à se modifier du fait de l'arrivée, au sein de l'avant-garde et de la SNM, des jeunes compositeurs issus de la classe de composition de Fauré au Conservatoire de Paris. L'intégration d'œuvres de Ravel, Koechlin, Schmitt ou Roger-Ducasse dans les programmes des concerts ${ }^{6}$ de la société est dans un premier temps facilitée par la position intermédiaire occupée par Fauré, qui est à la fois leur professeur de composition depuis 1896 et membre du comité de direction de la SNM depuis les années $1870^{7}$. D'ailleurs Schmitt, en 1925, s'en souviendra :

C'est [Fauré] [...] qui nous fit ouvrir toutes grandes, à Louis Aubert, Koechlin, [Roger] Ducasse, Grovlez, Ravel, Ladmirault, Eugène Cools, Enesco, à tous, les portes des concerts dominicaux, qui jusqu'alors ne s'entrebâillaient aux jeunes que si jalousement. Toute notre génération lui doit un tribut de reconnaissance (Schmitt 1925).

Peu à peu, et bien que Ravel et Schmitt fassent régulièrement partie du comité de direction de la SNM dans la décennie 1900, des tensions apparaissent au sein de l'avant-garde. Les anciens de la classe de Fauré commencent à contester, en privé

5 Le discours d'inauguration prononcé à la Schola Cantorum le 2 novembre 1900 synthétise de façon très éclairante le projet scholiste de subordination du musical au religieux ; voir Vincent d'Indy, La Schola cantorum. Son histoire depuis sa fondation jusqu'en 1925 (1927).

6 Voir notamment les programmes de la SNM entre 1895 et 1901 (Duchesneau 1997, p. 255-262).

$7 \quad$ Michel Duchesneau note que Chausson joue aussi un rôle de médiateur entre les élèves de Fauré et les Scholistes de la SNM. Mais son action sera moins durable que celle de Fauré puisqu'il meurt en 1899 (ibid., p. 35). 
comme dans la presse, la domination de d'Indy et des principes de création scholistes. Plusieurs événements viennent témoigner de ce conflit naissant : les Histoires naturelles de Ravel sont vivement critiquées par les Scholistes lors de leur création en 1907 à la SNM, puis Maurice Delage (alors élève de Ravel) et Koechlin se voient refuser l'exécution de leurs œuvres par le comité de direction de la SNM, comité dominé par les disciples de d'Indy ${ }^{8}$ (Duchesneau 1997, p. 37-38).

En s'inscrivant dans une double opposition à l'Institut et à la Schola, Ravel, Schmitt ou Koechlin sont amenés à prôner une esthétique plus radicalement autonome, c'est-à-dire dégagée des hétéronomies étatique et religieuse et excluant des problèmes d'ordre économique, éthique, politique du domaine musical. Cette position esthétique est bien une position "à faire " puisqu'elle n'existait pas dans les états structurels antérieurs du champ, et « ceux qui prétendent l'occuper ne peuvent la faire exister qu'en faisant le champ dans lequel elle pourrait trouver place, c'est-à-dire en révolutionnant un monde de l'art qui l'exclut, en fait et en droit " (Bourdieu [1992]1998, p. 131). Ainsi, ce contexte tendu débouche, en 1910, sur la création de la Société Musicale Indépendante ${ }^{9}$ - véritable objectivation institutionnelle du conflit -, société de concert dirigée par Ravel, Schmitt, Koechlin, Vuillermoz et quelques autres musiciens, dont le but est de s'émanciper de la mainmise de la SNM et de d'Indy sur la création musicale française. On a alors d'une part un espace qui voit l'Institut s'opposer à l'avant-garde, et d'autre part un sous-espace avant-gardiste (ou un " champ restreint ») qui, dégagé des principes académiques et économiques de création, voit les Indépendants s'opposer aux Scholistes (voir figure 2).

8 Ravel, dans une lettre de 1909 à Koechlin, parle d'un « comité, à l'exception de Schmitt, parfaitement homogène » (Ravel 1898, p. 101-102).

9 Que nous nommerons désormais SMI. 


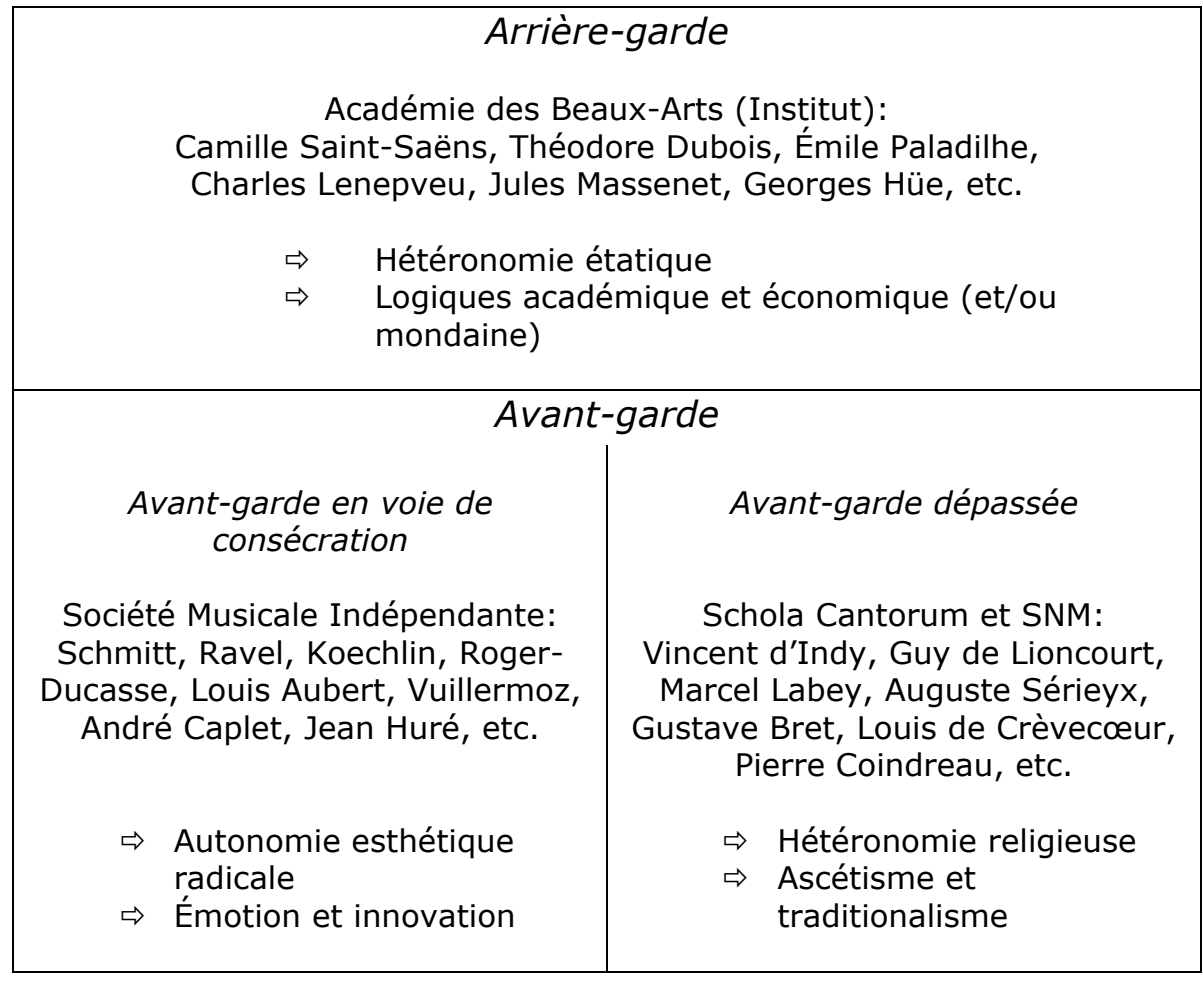

Figure 2 : Structure du champ musical français des années $1910^{10}$.

\section{Des Apaches Aux IndÉPENDANTS : LA CONSTRUCTION D'UNE LIGNÉE DEBUSSYSTE}

Là encore, chaque pôle ne représente qu'une tendance limite, et les frontières entre chaque pôle sont relativement souples, tout particulièrement au sein du sous-espace avant-gardiste. Certains compositeurs comme Déodat de Séverac, Albert Roussel, Gustave Samazeuilh ou Paul Ladmirault occupent des positions intermédiaires entre les deux pôles de l'avant-garde.

Outre leur position particulière au sein du champ musical français et leur double opposition à la Schola Cantorum et à l'Institut, quels facteurs ont bien pu favoriser le rapprochement des musiciens qui composent le comité de direction de la Société musicale indépendante en 1910 ? On ne peut manquer de souligner le fait que Ravel, Schmitt, Koechlin, Vuillermoz ou Roger-Ducasse ont tous fréquenté la classe de composition de Gabriel Fauré au Conservatoire de Paris entre 1896 et $1905^{11}$. Mais c'est surtout la constitution du groupe des «Apaches » qui peut nous intéresser ici, puisqu'en grande partie centrée sur l'œuvre de Claude Debussy.

10 Précisons que la structure du champ musical français est à peu près stable de 1910 à 1918 , mais qu'elle évolue au sortir de la Grande Guerre pour plusieurs raisons, dont l'affaiblissement du conflit entre Scholistes et Indépendants et le renouvellement de l'avant-garde avec l'arrivée des jeunes compositeurs de "l'école d'Arcueil » ou du " groupe des six ».

11 La configuration pédagogique particulière de la classe de Fauré n'est évidemment pas sans liens avec les manières de penser, de sentir et d'agir « mises en œuvre » par ses élèves dans les années 1900 et 1910 - Fauré est d'ailleurs élu, très symboliquement, président d'honneur de la sMi en 1910 (Nectoux 1975). Nous ne pouvons cependant développer trop longuement ici ce point ; voir Nectoux 1999. 
En 1902, un petit cercle d'amis commence à se former autour d'un enthousiasme communfaisantsuiteàlacréationdePelléasetMélisandeàl'Opéra-Comique.Rapidement baptisé groupe des "Apaches " ${ }^{12}$, il est constitué de poètes (Léon-Paul Fargue, Tristan Klingsor) ou de peintres (Édouard Bénédictus, Paul Sordes) mais surtout de musiciens (Maurice Ravel, Marcel Chadeigne, Florent Schmitt, Paul Ladmirault, Ricardo Viñes, Maurice Delage, Émile Vuillermoz, Déodat de Séverac et bien d'autres ${ }^{13}$ ), et se réunit régulièrement chez Paul Sordes, au 39 rue Dulong à Paris, puis tous les samedis chez Maurice Delage, dans un petit pavillon rue de Civry à Auteuil, afin de découvrir, de partager et de commenter des œuvres poétiques, littéraires et musicales : "Chaque semaine, l'un de nous avait quelque chose à lire, à déclamer, à faire entendre : un poème, une prose, un morceau » (Fargue 1939, p. 155).

Dès 1902, l'œuvre de Debussy est une des raisons principales du regroupement des jeunes artistes. On voit donc les Apaches, dans leurs pratiques musicales, réserver un temps important au jeu (et aux commentaires sur) des œuvres de Debussy ${ }^{14}$. Tristan Klingsor témoigne de cette présence debussyste : "Ricardo Viñes mettait toute la poésie de son toucher dans une pièce de Claude Debussy » (Klingsor 1939, p. 135). Et l'exercice du goût pour les œuvres de Debussy est affirmé avec encore plus de force et de précision par Léon-Paul Fargue : " [Nous] trempions corps et âme dans 1'“impressionnisme" de Claude Debussy » (Fargue 1939, p. 156).

Or, au début des années 1900, les œuvres de Debussy ne jouissent d'une reconnaissance unanime ni au sein du champ musical ni, a fortiori, au sein du public plus large. Un article écrit en 1905 par Émile Vuillermoz - sous le pseudonyme de Monsieur Lepic - en atteste :

Les debussystes ont remplacé les wagnériens dans les plaisanteries séculaires chères au public tardigrade. C'est sur eux que pleuvent depuis "Péléas » [sic] les brocarts et les railleries ! Rien n'est plus logique. Les adeptes d'un culte nouveau sont forcément suspects jusqu'au jour où leur divinité s'est solidement accréditée dans l'esprit des nations. Les wagnériens furent longtemps des types parfaits de grotesques : ils sont maintenant respectés et considérés car leur dieu a fait fortune. Les admirateurs de Debussy ne sont pas encore parvenus à cette période heureuse mais ils l'espèrent prochaine (Vuillermoz 1905b).

12 "Ce nom lui fut donné un dimanche où nous remontions la rue de Rome, après un concert, par un crieur de l'Intran[sigeant], qui nous bouscula sans malice : Attention les Apaches !" Le mot enchanta Vines qui lança ce jour-là l'Apachie dans l'Éternité » (Delage 1939, p. 99). Sur les Apaches, voir Pasler 1991 ; et surtout Haine 2006.

13 On remarque que la plupart, déjà, fréquentent ou ont fréquenté la classe de Fauré.

14 Pour être précis, ce sont ceux que les Apaches nomment «les Russes » qui complètent ce panthéon musical : Mili Balakirev, Alexandre Borodine, Alexandre Glazounov, Modeste Moussorgski et Nicolaï Rimski-Korsakov. 
Et l'on voit dans le même temps le rôle collectif d'apôtre que les Apaches choisissent d'endosser de manière relativement isolée ${ }^{15}$ (Pasler 2007).

On est alors peu surpris de constater que tout discours de Debussy, au sein du petit groupe des Apaches, est considéré comme hautement légitime (donc légitimant), permettant par exemple de faire contrepoids symbolique avec le verdict de l'Institut dans une lettre que Schmitt écrit en 1903 à Ravel après que ce dernier ait échoué au prix de Rome : "Consolez-vous, allez [...], le prix n'a plus longtemps à vivre. J'ai lu du bien de vous signé Debussy. Ça vaut tous les prix du monde » (Schmitt 1903). Chaque nouvelle œuvre du compositeur est également publiquement saluée et désignée comme un authentique événement musical :

La musique semble morte à Paris en cette arrière-saison [...]. À part La Mer de Debussy, splendide triptyque impressionniste exposé chez Chevillard, aucune œuvre sérieuse n'a été offerte en pâture à l'avidité des musicographes (Vuillermoz 1905a).

Le groupe des Apaches représente donc une étape intermédiaire entre la classe de Fauré et la création de la SMI, durant laquelle "l'exercice ${ }^{16}$ " du goût pour la musique de Debussy occupe une place fondamentale.

\section{La SMI et l'œuvre de Debussy comme point de départ}

Avec la création de la SMI en 1910, les Indépendants peuvent désormais mettre en œuvre beaucoup plus efficacement leur entreprise de valorisation en intégrant aux programmes de leurs concerts la musique de Debussy. Cette dernière est ainsi jouée à cinq des douze premiers concerts ${ }^{17}$ de 1910 et 1911, le compositeur venant même interpréter en personne Danseuses de Delphes, Voiles, La cathédrale engloutie et La danse de Puck, quatre de ses Préludes pour piano, au concert du 25 mai 1910 (Duchesneau 1997, p. 305-307). On assiste alors à un léger glissement : en associant symboliquement ses œuvres aux leurs, les Indépendants célèbrent Debussy mais, surtout, le proclament explicitement fondateur de leur lignée collective.

Cette interprétation est confortée par certaines de leurs déclarations. Ravel affirme par exemple en 1912 que Debussy est « le plus profondément musical des compositeurs d'aujourd'hui » (Ravel 1989, p. 301), puis écrit l'année suivante, en soulignant le clivage sMI/Schola : "Actuellement, deux écoles sont en présence : l'ancienne est constituée par les disciples de César Franck. M. Claude Debussy est considéré à juste titre comme le principal initiateur de la nouvelle " (Ravel 1989, p. 311). De même, pour Koechlin, en 1916, la création de la smi est :

15 Le succès progressif de Pelléas, en termes d'affluences, obéit en partie à des logiques externes au champ musical, et relativement spécifiques au genre de l'opéra. Jann Pasler en esquisse une analyse (1987).

16 Au double sens du terme, c'est-à-dire de "l'entraînement qui exerce » et de « la faculté qu'on exerce » (Hennion 2003, p. 300).

17 Et même six, si l'on prend en compte le pastiche À la manière de Claude Debussy d'Alfredo Casella au concert du 6 mars 1911. 
Logique, car il n'était pas naturel que les héritiers de la tradition musicale de Chabrier, de Fauré et de Debussy, dépendissent d'une majorité essentiellement " d'indyste ». [...] Les Debussystes de la Société Nationale (d'ailleurs en minorité) se sentaient de plus en plus loin des « scholistes » (Koechlin 2006, p. 99-101).

Et l'on voit bien comment l'œuvre de Debussy apparaît aux Indépendants à la fois comme une ressource musicale et esthétique mobilisable dans la lutte contre la Schola, et comme un instrument de définition de soi ${ }^{18}$.

Bref, dire que les Indépendants s'approprient l'œuvre de leur aîné, ce n'est donc pas seulement décrire l'exercice d'un goût par un processus de réception active. C'est dans le même temps indiquer qu'ils s'en emparent symboliquement, qu'ils s'en réclament, qu'ils le revendiquent. Car à travers leurs oppositions à l'Institut et surtout à la Schola, l'élaboration de leur identité esthétique collective s'appuie fermement sur l'œuvre de Debussy qui devient une référence indiscutée, un support nécessaire, une fondation inébranlable ${ }^{19}$. C'est par cette double action, qui consiste à la fois à faire de l'auteur de Pelléas l'ancêtre originel de leur lignée artistique et à lutter (avec succès) pour exister au sein du champ musical, que grandit peu à peu l'œuvre de Debussy.

\section{Les INDÉPENDANTS À L’ÉCOUTE DE DeBussy}

Les Apaches/Indépendants goûtent les œuvres de Debussy, certes. Mais comment les goûtent-ils ? Quelles logiques sont précisément au fondement de leur admiration pour Pelléas et Mélisande, La mer, les Images pour orchestre ou les Préludes pour piano? En gardant en tête la structure du champ musical telle que nous l'avons restituée, nous pouvons opérer un "gros plan » sur les écrits de Schmitt, Ravel, Koechlin ou Vuillermoz en les considérant comme des outils dont les deux fonctions indissociables sont l'explicitation et la légitimation ${ }^{20}$ du goût pour la musique de Debussy.

18 Une critique musicale de Schmitt va dans le même sens : « Au Châtelet, M. Vincent d'Indy, pour la seconde fois, dirigeait son noble et ardent Jour d'été à la montagne, tandis que M. Gabriel Pierné, dans une ingénieuse et louable intention de symétrie - ou de compensation - exécutait les Trois Nocturnes, de M. Claude Debussy, qui sont, avec l'Après-midi d'un Faune et les Jeux de vagues, du même auteur, parmi les musiques les plus marquantes et les plus séduisantes de ces trois derniers siècles et sans doute aussi de nombreux siècles futurs, œuvres d'ailleurs célèbres et universellement admirées " (Schmitt 1912). On ne peut que souligner ici le fait que le point de vue et les écrits (innombrables) de Florent Schmitt sont souvent ignorés des musicologues et des historiens travaillant sur la musique française du début du $\mathrm{xx}^{\mathrm{e}}$ siècle. Sa personnalité sulfureuse et provocatrice, son appartenance au groupe «Collaboration » en tant que président d'honneur, au début des années 1940, ainsi que sa condamnation par le Comité national d'épuration des gens de lettres, auteurs et compositeurs en 1945, en fourniraient-elles des éléments d'explication?

19 Déodat de Séverac, qui présente la particularité d'avoir à la fois participé aux réunions des Apaches et d'avoir été un élève assidu de la Schola, souligne dès 1907 dans sa thèse de fin d'études à la Schola (publiée en 1908) le clivage entre une chapelle « de droite » qui désigne la Schola et dont le leader est d'Indy, et une chapelle « de gauche " qui désigne les Apaches et dont le leader symbolique est Debussy, pour les renvoyer dos à dos (Séverac 1908). Sur ce conflit entre " d'indystes » et « debussystes ", voir Michel 2000. Voir également Fulcher 1999, sur l'idée de chapelles comme point de référence pour l'époque.

20 La fonction de légitimation (ou de dé-légitimation) de la critique musicale et son impact sur les carrières des compositeurs ont été étudiés par Esteban Buch dans son ouvrage Le cas Schoenberg. Naissance 
La lutte des Indépendants contre les Scholistes se cristallise principalement autour de deux rejets : rejet de l'ascétisme catholique et rejet de l'attachement aux traditions et aux règles, soit deux principes éthiques que d'Indy et la Schola importent, plus ou moins consciemment, dans la pratique de la composition. À l'ascétisme et au traditionalisme sont alors respectivement opposées logique de l'émotion et logique de l'innovation, dont Debussy est peu à peu désigné comme le champion.

\section{Logique de l'émotion}

En premier lieu, ce qui est rejeté dans l'ascétisme ce n'est pas tant la régularité et le sérieux de l'effort créateur, ni même l'exigence du travail technique qui permet d'acquérir le " métier ». C'est bien plus une certaine sécheresse d'inspiration, une rigidité perçue dans le maniement du matériau musical ${ }^{21}$. La pensée esthétique des Indépendants, imprégnée de ce dégoût du rigide et de l'austère, s'organise alors autour d'un clivage très net entre le cérébral et le sensible, l'inhumain et l'humain, le cerveau et le cœur, la raison et la passion, la sécheresse et l'émotion, le glacé et le brûlant, etc. $\mathrm{Et}$, dans chacune de ces oppositions binaires, le premier terme est systématiquement dénigré au profit du second. À travers la lutte esthétique face à la schola, la conception romantique de la musique comme outil spécifique d'expression des sentiments et des émotions est vigoureusement réinvestie et l'opposition cérébralité/sensibilité devient une catégorie de perception profondément structurante ${ }^{22}$.

Les œuvres de Debussy deviennent alors de véritables remparts contre ce qui est perçu comme un excès de raison ou de froide réflexion, et sont associées au registre émotionnel ou sensible de manière extraordinairement récurrente. Dès 1905, Émile Vuillermoz, dont l'activité débordante de critique musical en fait le véritable " porte-plume » des Apaches puis des Indépendants, déclare : "Le Prélude à l'" Après-midi d'un Faune " [sic] de Debussy nous a, une fois de plus, roulé dans le torrent des sensations divines!" (Vuillermoz 1905b). Même son de cloche chez Ravel qui, dans un article de 1913 défendant les Images pour orchestre contre les attaques répétées de Gaston Carraud, ne manque pas d'avouer se sentir étreint : "jusqu'aux larmes par cette ruisselante Iberia, par ces Parfums de la nuit, si profondément émouvants » (Ravel 1989, p. 313). Dans une conférence prononcée en 1916, Charles Koechlin souligne lui aussi, à propos de Pelléas et Mélisande, «l'émotion profonde de cet art admirable » (Koechlin 2006, p. 69). La formule ne peut pas être plus claire ; le drame lyrique de Debussy est associé au registre émotionnel, et c'est ce

de l'avant-garde musicale (2006). Sur la critique musicale française durant la période qui nous intéresse, voir Goubault 1984.

21 Schmitt, à propos de l'opéra Fervaal de d'Indy, parle de " renoncement " et de " mortification catholique » (Schmitt 1913a).

22 C'est ainsi que l'on peut également comprendre la mise en avant du paramètre harmonique (verticalité) chez les Indépendants comme une protestation face à l'usage appuyé d'un contrepoint rigoureux (horizontalité) chez d'Indy et ses disciples, interprété comme une retraduction de l'ascétisme catholique dans le maniement même du matériau musical ; voir l'article d'Émile Vuillermoz, "La Schola et le Conservatoire» (1909). 
qui le rend admirable.

Mais on trouve sans doute l'exemple le plus éclatant de cette apologie émotionaliste de l'œuvre de Debussy dans une critique de Florent Schmitt de 1913 consacrée là encore aux Images pour orchestre:

Et qu'oser dire de ces Images qui ne soit une profanation ? L'admiration devant les grandes œuvres de la nature ou des hommes n'est-elle pas le plus souvent muette ? La musique de M. Claude Debussy [...] est de celles qui vous déconcertent au point de vous dégoûter, pour de longs jours, de toutes les autres, y compris et surtout de la vôtre, si vous avez l'infortune d'en commettre. Pour le moment, je suis sous le coup d'une émotion trop forte, que je ne voudrais pas troubler par l'analyse, et les mots, pas plus que les sons, ne sauraient me venir. Je vous demanderai donc de me faire crédit de quelques jours. Grâces soient rendues à Claude Debussy (Schmitt 1913a).

On voit que le registre du ressenti "brut ", des sensations, des émotions, est très nettement opposé au registre de l'analyse et de la réflexion. Pour bien mettre en évidence la "nature émotionnelle » de ces Images pour orchestre, le procédé de Schmitt consiste à refuser purement et simplement la critique musicale, elle-même associée à la logique cérébrale et désignée ennemie de l'œuvre. Et, conjointement à cette mise en avant du registre émotionnel, est déployée une puissante rhétorique de la valorisation, les analogies religieuse et juridique qui parsèment le texte étant à prendre au sérieux. Car si Schmitt parle de " profanation » des Images par son discours de critique, et pense lui-même " commettre » de la musique, c'est parce qu'il se place face à l'œuvre de Debussy comme un croyant face à un objet sacré ou un individu face à un texte de loi. Il fait ainsi de Debussy l'équivalent, dans le domaine musical autonomisé, du dieu ou du juge, c'est-à-dire de l'autorité absolue et indépassable.

\section{Logique de l'innovation}

En second lieu, la pensée des Indépendants, sous des contours évolutionnistes, laisse apparaître une préoccupation constante de l'avenir musical, un intense désir de dépassement ; d'Indy, à l'opposé, préconise une musique du présent qui s'inscrive fermement dans une tradition tout en la renouvelant, qui ne s'écarte pas des voies que les grands maîtres du passé lui ont tracée ${ }^{23}$. Dès lors, on comprend aisément que la notion de « progrès » sous-tende nombre d'écrits de Vuillermoz (Trottier 2014) :

Si l'on admet en art la notion du progrès [...] il faut donc le faire résider dans le perfectionnement du langage, dans l'amélioration de l'outillage des forgerons harmonieux. [...] Le musicien qui apporte à ses contemporains des trouvailles de mots, des expressions heureuses, une écriture d'une richesse et d'une ingéniosité insoupçonnées est [...] un grand bienfaiteur de son art.

23 L'enseignement de la composition nécessite ainsi, pour d'Indy, une étude approfondie des procédés compositionnels des différentes périodes de l'histoire de la musique occidentale (d'Indy 1912b). 
C'est un « éveilleur ». Il engendre des chefs-d'œuvre, il ouvre des portes que franchiront demain des artistes de génie qui cherchaient leur voie. Il faut saluer la naissance de ces explorateurs comme une rare libéralité du destin (Vuillermoz 1923, p. 5-6).

Schmitt, dont l'oreille semble elle aussi obsédée par l'inconnu, le nouveau sonore, le moderne, l'inoui, ne cesse de mettre en œuvre son amour de l'innovation dans ses nombreux articles de critique musicale, écrivant par exemple en 1918, à propos des Pagine di guerra d'Alfredo Casella :

Il s'agit de musique, de musique dans l'acception la plus noble et la plus désintéressée, une musique qui, rompant une fois pour toutes avec un passé sans gloire, part résolument du présent [...] pour s'élancer audacieusement vers l'inconnu [...] (Schmitt 1918).

Et c'est au travers de cette " disponibilité à s'engager avec ce qui n'est pas encore classé, admis, subsumé sous des catégories fixes ", pour reprendre les mots d'Adorno ([1994]2009, p. 170), qu'il faut comprendre la remarque de Koechlin selon laquelle Debussy est de ceux qui « ont déterminé des mouvements nouveaux et marqué des points de repère » (Koechlin 2006, p. 65). Vuillermoz, enfin, livrera le bilan suivant à la mort de Debussy:

On ne saurait en quelques lignes hâtives caractériser l'art de Debussy et faire l'inventaire de ce qu'il apporta de nouveau dans notre musique. Il fut, essentiellement, un briseur de chaînes et un libérateur (Vuillermoz 1918).

À travers cette tension collective vers l'innovation - indissociable de la logique de l'émotion - les Indépendants font donc de Debussy un novateur, un éclaireur leur indiquant le chemin à suivre entre les ténèbres de l'institut et celles de la Schola ${ }^{24}$.

\section{CONCLUSION}

On voit ainsi comment, dans les années 1900 et 1910, les Indépendants contribuent grandement à « faire » la valeur de l'œuvre de Debussy en le « saisissant » - au double sens du terme, c'est-à-dire en s'en emparant et en la comprenant - au travers d'une double esthétique de l'émotion et de l'innovation. La restitution de la structure et de l'évolution du champ musical français de la fin du XIX siècle et du début du Xx ${ }^{\mathrm{e}}$ siècle s'avère indispensable, en ce qu'elle nous permet de réaliser que cette " saisie » trouve sa source dans un contexte macro-social de lutte(s) indissociablement institutionnelle(s), esthétique(s) et musicale(s), notamment face à la Schola Cantorum de Vincent d'Indy.

Certes, dès les années 1890, d'autres figures du champ musical français ont cherché

24 Notons que c'est le timbre qui, aux côtés de l'harmonie, est régulièrement désigné comme le paramètre le plus révélateur de l'innovation debussyste : «Debussy, magicien des sonorités orchestrales, est aussi un extraordinaire poète du clavier, un miraculeux prestidigitateur, qui sait, à son gré pour les besoins de son art, transformer un piano en orgue, en luth ou en harpe éolienne » (Vuillermoz 1911). 
à promouvoir l'œuvre de Debussy en saluant l'originalité du Prélude à l'après-midi d'un faune, du Quatuor ou des Nocturnes - au premier rang desquelles on trouve, bien sûr, ses amis Louis Laloy (Priest [1999]2007) et Paul Dukas (1948). Mais si l'appropriation de l'œuvre de Debussy par les Indépendants est si décisive, c'est parce que tout en étant parmi les premiers à faire de Debussy un jalon indiscutable de l'histoire de la musique ${ }^{25}$, ils s'en réclament de façon cohérente et quasi organisée pour fonder leur propre existence artistique. Et, outre que la SMI constitue peu à peu l'une des positions collectives les plus en vue du champ musical français, certains de ses membres accèderont, dans les décennies suivantes, à des positions plus prestigieuses encore ${ }^{26}$, continuant de faire de Debussy un véritable " classique $^{27}$ ". L'entreprise collective des Indépendants se rapproche ainsi d'une petite "révolution symbolique » (Bourdieu 1987, p. 177), c'est-à-dire d'une imposition décisive, au sein du champ musical, de catégories de perception et d'appréciation transformant l'appréhension de l'œuvre debussyste.

Notre objectif n'est évidemment pas de réduire la valeur de cette dernière. Il est, beaucoup plus simplement, de mettre en évidence les conditions sociales et historiques favorables dont elle a en grande partie bénéficié, et dont sa saisie par les Indépendants dans les années 1900 et 1910 participe ${ }^{28}$, le tout dans un refus de céder à une vision téléologique de l'histoire de la musique.

Pour valider empiriquement cette approche, il faudrait bien entendu la compléter et la prolonger en enquêtant sur les différentes étapes antérieures de l'appropriation de l'œuvre de Debussy au sein du champ musical français, mais aussi sur les étapes ultérieures de sa canonisation dont l'entre-deux-guerres constitue un moment décisif comme les travaux de Barbara Kelly (2008), Marianne Wheeldon (2010) et Danick Trottier (2014) tendent à en faire la démonstration. À cela s'ajoutent les enjeux de réception liés aux publics plus " profanes ", en passant par d'autres contextes nationaux, etc. Le chantier de recherche alors ouvert serait immense mais passionnant.

25 Ravel, lors d'une conférence prononcée aux États-Unis en 1928, dira de Debussy qu'il est « le plus extraordinaire génie de l'histoire de la musique française » (Ravel 1989, p. 53), ce qui n'est pas sans lien avec le travail de canonisation opéré par Vuillermoz (Trottier 2014).

26 Outre Maurice Ravel qui n'occupera jamais de poste officiel mais dont la carrière exceptionnelle en fera une personnalité très écoutée, Florent Schmitt accèdera à la direction du Conservatoire de Lyon de 1921 à 1924, sera nommé membre de l'Institut en 1936 et se verra confier la très convoitée chronique musicale du Temps entre 1929 et 1939 ; Charles Koechlin aura de nombreux élèves tels que Sauguet, Poulenc ou Tailleferre peu après 1918, enseignera à la nouvelle Schola Cantorum à partir de 1937 et publiera un influent traité d'orchestration (Duchesneau 2006). Signalons enfin que Vuillermoz et Koechlin publieront chacun une monographie sur Debussy (Vuillermoz [1957]1962 ; Koechlin 1927).

27 C'est toute l'ambition argumentative de Charles Koechlin dans un article de 1927 : « Ce mot de classique, s'il n'est pas qu'illusion, ou qu'artifice de professeur, je ne le puis définir qu'avec les termes exposés plus haut, et relatifs surtout à la beauté du style, à sa pureté. Alors Debussy et Fauré en sont dignes, autant que Mozart » (Koechlin 2006, p. 68).

28 Si nous privilégions ici le versant " constructiviste " du processus, nous n'en nions pas pour autant le versant "réaliste ». En effet, l'œuvre de Debussy, comme toute partie du réel, présente une certaine " résistance » aux multiples appréhensions qui peuvent en être faites. Ce n'est donc évidemment pas un hasard si c'est cet œuvre-là que les Indépendants s'approprient et non un autre. 


\section{BIBLIOGRAPHIE}

Adorno, Theodor W. ([1994]2009), Introduction à la sociologie de la musique, traduit de l'allemand par Vincent Barras et Carlo Russi, Genève, Contrechamps.

Bandier, Norbert (1999), Sociologie du surréalisme 1924-1929, Paris, La Dispute.

Boltanski, Luc (1975), « La constitution du champ de la bande dessinée », Actes de la recherche en sciences sociales, vol. $1, \mathrm{n}^{\circ} 1, \mathrm{p} .37-59$.

Bourdieu, Pierre (1979), La distinction. Critique sociale du jugement, Paris, Minuit.

Bourdieu, Pierre (1987), Choses dites, Paris, Minuit.

Bourdieu, Pierre ([1992]1998), Les règles de l'art. Genèse et structure du champ littéraire, Paris, Seuil.

Bowness, Alan ([1989]2011), Les conditions du succès, traduit de l'anglais par Catherine Wermester, Paris, Allia.

Buch, Esteban (2006), Le cas Schoenberg. Naissance de l'avant-garde musicale, Paris, Gallimard.

Delage, Maurice (1939), «Les premiers amis de Ravel », dans Colette et al., Maurice Ravel par quelquesuns de ses familiers, Paris, Tambourinaire, p. 97-114.

Duchesneau, Michel (1997), L'avant-garde musicale à Paris de 1871 à 1939, Liège, Mardaga.

Duchesneau, Michel (2006), "Introduction », dans Charles Koechlin, Écrits, "Vol.1. Esthétique et langage musical ", textes réunis et présentés par Michel Duchesneau, Spirimont, Mardaga, p. 9-36.

Dukas, Paul (1948), Écrits sur la musique, Paris, SEFI.

Fabiani, Jean-Louis (1993), "Sur quelques progrès récents de la sociologie des œuvres ", Genèses, vol. $11, \mathrm{n}^{\circ} 1$, p. 148-167.

Fabiani, Jean-Louis ([1999]2001), "Les règles du champ », dans Bernard Lahire (dir.), Le travail sociologique de Pierre Bourdieu. Dettes et critiques, Paris, La Découverte, p. 75-91.

Fargue, Léon-Paul (1939), "Autour de Ravel », dans Colette et al., Maurice Ravel par quelques-uns de ses familiers, Paris, Tambourinaire, p. 153-162.

Fulcher, Jane (1999), French Cultural Politics and Music. From the Dreyfus Affair to the First World War, Oxford/New York, Oxford University Press.

Gamboni, Dario (1989), La plume et le pinceau. Odilon Redon et la littérature, Paris, Minuit.

Goubault, Christian (1984), La critique musicale dans la presse française de 1870 à 1914, Genève, Slatkine.

Haine, Malou (2006), "Cipa Godebski et les Apaches », Revue belge de musicologie, vol. 60, p. 221266.

Hennion, Antoine (2003), "Ce que ne disent pas les chiffres. Vers une pragmatique du goût », dans Olivier Donnat et Paul Tolila (dir.), Le(s) public(s) de la culture, Paris, Presses de Sciences-Po, p. 287204.

D’Indy, Vincent (1895), « L'institut vu par ceux qui n'en sont pas », Le Figaro (28 octobre).

D’Indy, Vincent (1912a), «L'institut », Le Temps (24 octobre).

D'Indy, Vincent (1912b), Cours de composition musicale. Premier livre, rédigé avec la collaboration d'Auguste Sérieyx, Paris, Durand et fils.

D'Indy, Vincent (1927), La Schola cantorum. Son histoire depuis sa fondation jusqu'en 1925, Paris, Bloud et Gay.

Kelly, Barbara L. (2008), « Debussy and the Making of a musicien français. Pelléas, the Press, and World War I », dans Barbara Kelly (dir.), French Music, Culture, and National Identity, 1870-1939, Rochester, University of Rochester Press, p. 58-76. 
Klingsor, Tristan (1939), "L'époque Ravel », dans Colette et al., Maurice Ravel par quelques-uns de ses familiers, Paris, Tambourinaire, p. 125-140.

Koechlin, Charles (1927), Debussy, Paris, Laurens.

Koechlin, Charles (2006), Écrits, «Vol. 1. Esthétique et langage musical», textes réunis et présentés par Michel Duchesneau, Spirimont, Mardaga.

Lahire, Bernard ([1999]2001), "Champ, hors-champ, contrechamp », dans Bernard Lahire (dir.), Le travail sociologique de Pierre Bourdieu. Dettes et critiques, Paris, La Découverte, p. 23-57.

Michel, Fabien (2000), «La querelle des d'indystes et des debussystes », Thèse de doctorat, Université de Dijon.

Nectoux, Jean-Michel (1975), "Ravel/Fauré et les débuts de la Société Musicale Indépendante ", Revue de musicologie, vol. 61, nº 2, p. 295-318.

Nectoux, Jean-Michel (1999), " "Tous écoutent la parole du maître". Gabriel Fauré et ses élèves ", dans Anne Bongrain et Alain Poirier (dir.), Le conservatoire de Paris. Deux cents ans de pédagogie, 17951995, Paris, Buchet/Chastel, p. 345-360.

Pasler, Jann (1987), "Pelléas and Power. Forces behind the Reception of Debussy's Opera ", 19thCentury Music, vol. 10, n 3, p. 243-264.

Pasler, Jann (1991), « La Schola Cantorum et les Apaches. L'enjeu du pouvoir artistique, ou Séverac médiateur et critique », dans Hugues Dufourt et Joël-Marie Fauquet (dir.), La musique. Du théorique au politique, Paris, Klincksieck, p. 313-343.

Pasler, Jann (2007), «A Sociology of les Apaches. Sacred Battalion for Pelléas», dans Barbara Kelly et Kerry Murphy (dir.), Berlioz and Debussy. Sources, Contexts and Legacies, London, Ashgate, p. 148-166.

Priest, Deborah ([1999]2007), Debussy, Ravel et Stravinski. Textes de Louis Laloy (1874-1944), Paris, L'Harmattan.

Ravel, Maurice (1989), Lettres, écrits, entretiens, présentés et annotés par Arbie Orenstein, Paris, Flammarion.

Saint-Arroman, Gilles (2011), "Les écrits de Vincent d'Indy, d'une pensée à sa mise en acte ", Thèse de doctorat, Université Paris-Sorbonne.

Schmitt, Florent (1903), Lettre autographe signée à Maurice Ravel, 11 juillet 1903, Londres, Catalogue de Vente Drouot-Richelieu, 26 juin 2000, nº 68, p. 37.

Schmitt, Florent (1912), « Les concerts », La France (22 octobre).

Schmitt, Florent (1913a), « Les concerts », La France (21 janvier).

Schmitt, Florent (1913b), «Les concerts », La France (28 janvier).

Schmitt, Florent (1918), "Les concerts », La France (6 mars).

Schmitt, Florent (1925), «Les arts et la vie. Sur Gabriel Fauré », La revue de France (1 ${ }^{\text {er }}$ janvier).

Schwartz, Manuela (dir.) (2006), Vincent d'Indy en son temps, Liège, Mardaga.

Séverac, Déodat de (1908), "La centralisation et les petites chapelles musicales », Le courrier musical ( $1^{\text {er }}, 15$ janvier et $1^{\text {er }}$ mars).

Strasser, Michael C. (1998), «Ars Gallica. The Société Nationale de Musique and its Role in French Musical Life, 1871-1891 », Thèse de doctorat, University of Illinois.

Trottier, Danick (2014), «Creating a Canon. Émile Vuillermoz and French Musical Modernism », dans Barbara Kelly et Christopher Moore (dir.), Musical Criticism in France Interwar Period, Cambridge, Cambridge University Press.

Vuillermoz, Émile (1905a), «Lettre parisienne. La vie musicale», sous le pseudonyme de Monsieur Lepic, Le Monde lyonnais (19 novembre).

Vuillermoz, Émile (1905b). «Lettre parisienne. La vie musicale », sous le pseudonyme de Monsieur Lepic, Le Monde lyonnais (3 décembre). 
Vuillermoz, Émile (1909), «La Schola et le Conservatoire », Le Mercure de France (16 septembre).

Vuillermoz, Émile (1911), « Bloc-notes du mélomane », Paris-Midi (29 mars).

Vuillermoz, Émile (1914), « La musique au concert », Comcedia (9 mars).

Vuillermoz, Émile (1918), « Claude Debussy est mort », L'Éclair (26 mars).

Vuillermoz, Émile (1923), Musiques d'aujourd'hui, Paris, G. Crès et Cie.

Vuillermoz, Émile ([1957]1962), Claude Debussy, Paris, Flammarion.

Wheeldon, Marianne (2010), "Debussy's Legacy. The Controversy over the Ode à la France ", The Journal of Musicology, vol. 27, n 3, p. 304-341. 